\title{
TEMPERATURE BOUNDS ON THE INFINITE ROD
}

\section{DANIEL RESCH}

The temperature of an infinite rod is measured at $n$ points $x_{i}$ at the respective times $t_{i}$ and the temperatures are denoted by $c_{i}$, $i=1,2, \cdots, n$. On the basis of this data, what will the bounds on the temperature be at any point $x$ of the rod and at any time $t$ ? A theorem of P. C. Rosenbloom leads to a satisfactory theoretical solution of the problem as follows. ${ }^{1}$ Consider the $n+1$ functions $f_{1}(y), \cdots, f_{n}(y), f(y)$, continuous on $(-\infty, \infty)$, and the numbers $c_{1}, \cdots, c_{n}$. Let $M$ be the class of all nondecreasing functions $\alpha(y)$ such that

$$
\int_{-\infty}^{\infty} f_{i}(y) d \alpha(y)=c_{i}, \quad i=1,2, \cdots, n .
$$

Then the extremum of the integral

$$
\int_{-\infty}^{\infty} f(y) d \alpha(y)
$$

for $\alpha(y) \in M$ will be attained, in general, when $\alpha(y)$ is a step function of at most $n$ jumps. The application to the problem at hand follows as a consequence of a theorem of D. V. Widder: $:^{2}$ if $u(x, t)$ is a nonnegative solution of the heat equation, $\delta u=u_{x x}-u_{t}=0$, then it can be represented as

$$
u(x, t)=\int_{-\infty}^{\infty} k(y-x, t) d \alpha(y)
$$

where $k(z, t)=(4 \pi t)^{-1 / 2} \exp \left(-z^{2} / 4 t\right)$ and $\alpha(y)$ is nondecreasing. Hence the substitutions $f_{i}(y)=k\left(y-x_{i}, t_{i}\right), u\left(x_{i}, t_{i}\right)=c_{i}, i=1,2, \cdots$, $n$, and $f(y)=k(y-x, t)$ reduce the problem to that of finding the jumps of the extremizing function $\alpha(y)$.

For $n=1$, a solution is readily obtained. But for larger $n$, the algebraic equations involved become so highly encumbered that explicit solutions are not obtainable. The problem for $n=1$ can also be solved with less high-powered methods. It is shown that if the tempera-

Presented to the Society, April 28, 1951; received by the editors November 28' 1951.

1 P. C. Rosenbloom, Quelques classes de problème extrémaux, Bull. Soc. Math. France vol. 79 (1951) pp. 1-58.

2 D. V. Widder, Positive temperatures on an infinite rod, Trans. Amer. Math. Soc. vol. 55 (1944) pp. 85-95. 
ture is known at one point $\left(x_{1}, t_{1}\right)$, then the temperature will be bounded from above at any previous time and from below at any fu'ture time at every point of the rod. Theorem 2 of this note uses the result to prove an analogue of Harnack's second convergence theorem for a monotone sequence of harmonic functions bounded at one point of a domain.

LEMma. Consider the integrals

$$
I=\int_{-\infty}^{\infty} f(y) d \alpha(y), \quad J=\int_{-\infty}^{\infty} g(y) d \alpha(y)
$$

with $f(y), g(y)$ continuous, $f(y) \neq 0, g(y)<\infty$, and $\alpha(y)$ nondecreasing. Let $h(y)=g(y) / f(y)$. Then if $L=\inf _{-\infty<y<\infty} h(y)$ and $K=\sup _{-\infty<y<\infty} h(y)$, it follows that $L I \leqq J \leqq K I$.

Proof. For the last inequality, $J=\int_{-\infty}^{\infty} g(y) d \alpha(y)=\int_{-\infty}^{\infty} h(y) f(y) d \alpha(y)$ $\leqq \sup _{-\infty<y<\infty} h(y) \int_{-\infty}^{\infty} f(y) d \alpha(y)=K I$. The proof that $J \geqq L I$ is similar.

It is interesting to note that the values for $L$ and $K$ are the best possible in the inequalities. For we can find a value $y_{0}$ such that $h\left(y_{0}\right)>K-\epsilon$ for any $\epsilon>0$. Then if we choose for $\alpha(y)$ the function

$$
\alpha_{0}(y)=\left\{\begin{array}{l}
0,-\infty<y<y_{0} \\
1, y_{0} \leqq y<\infty
\end{array}\right.
$$

it follows that $I=f\left(y_{0}\right)$ and $J=g\left(y_{0}\right)$. Now if there exists a value $K_{1}$ with $K_{1}<K$ and $J \leqq K_{1} I$, then for $\alpha_{0}(y),(K-\epsilon) I<J \leqq K_{1} I$ whence by the arbitrariness of $\epsilon, K<K_{1}$, an obvious contradiction. That $L$ is also the best possible value is shown similarly.

TheOREM 1. If $u(x, t)$ represents a non-negative solution of the heat equation and $u\left(x_{1}, t_{1}\right)=c_{1}$, then for $0<t<t_{1}, u(x, t) \leqq c_{1} H(x, t)$ and for $t>t_{1}>0, u(x, t) \geqq c_{1} H(x, t)$, where

$$
H(x, t)=\left(t_{1} / t\right)^{1 / 2} \exp \left[-\frac{\left(x-x_{1}\right)^{2}}{4\left(t-t_{1}\right)}\right] .
$$

Proof. The results follow from the lemma with $f(y)=k\left(y-x_{1}, t_{1}\right)$ and $g(y)=k(y-x, t)$. Then $I=c_{1}$ and $J=u(x, t)$. We seek the infimum and supremum of the function $h(y)=k(y-x, t) / k\left(y-x_{1}, t_{1}\right)$. With the aid of the calculus it is found that

$$
c_{1} H(x, t)= \begin{cases}\inf _{-\infty<\nu<\infty} h(y), & \text { for } 0<t<t_{1}, \\ \sup _{-\infty<\nu<\infty} h(y), & \text { for } t>t_{1}>0 .\end{cases}
$$


Although the method cannot be extended for predicting temperature bounds on the basis of known temperatures at more than one point, it can be directly applied to finding the bounds on the temperature gradient.

THEOREM 2. If $\left\{u_{n}(x, t)\right\}$ is a monotone increasing sequence of nonnegative solutions of the heat equation $(n=1,2, \cdots)$, and the sequence is bounded at one point $\left(x_{1}, t_{1}\right)$ of the upper half $(x, t)$ plane, then the sequence converges uniformly in region $\left|x-x_{1}\right| \leqq A\left(t_{1}-t\right)^{1 / 2}, t \geqq \epsilon$, for arbitrary but positive $A, \epsilon$. That is, the region is enclosed above the $t$ axis by any parabola having vertex at $\left(x_{1}, t_{1}\right)$ and axis $x=x_{1}$.

Proof. Let $D_{m n}(x, t)=u_{m}(x, t)-u_{n}(x, t), m>n$. Then $D_{m n}(x, t)$ $\geqq 0$ and $\delta D_{m n}(x, t)=0$. By Theorem $1, D_{m n}(x, t) \leqq D_{m n}\left(x_{1}, t_{1}\right) H(x, t)$ for $0<t<t_{1}$ and all $x$. But since the sequence converges at $\left(x_{1}, t_{1}\right)$, then given a $\tau>0$, there exists a number $M(\tau)$ such that $0 \leqq D_{m n}(x, t)$ $<\tau$ for $m, n>M(\tau)$. Now take $m$ and $n$ sufficiently large that 0 $\leqq D_{m n}\left(x_{1}, t_{1}\right)<\tau[H(x, t)]^{-1}$. This is permissible since $H(x, t)$ is finite and nonzero in the described region. But then $0 \leqq D_{m n}(x, t)<\tau$ for $m, n>M_{1}(\tau)>M(\tau)$. This proves the theorem.

UNIVERSITY OF MichIGAN 